THE MILLIMETER ARRAY

\author{
ROBERT L. BROWN
}

National Radio Astronomy Observatory, Edgemont Road, Charlottesville, Virginia 22903, USA

\title{
GENESIS OF THE MMA
}

In recognition of the achievements and the potential of scientific research at millimeter wavelengths, the NSF in 1983 formed a subcommittee chaired by Professor Alan Barrett, to offer guidance on the priorities for future development of millimeter and submillimeter astronomy. Among the committee's three principal recommendations was a request for a design study for a national millimeter-wavelength synthesis array. This particular recommendation was an encouragement that the NSF build on the seminal interferometric millimeter work being done with the Owens Valley Radio Observatory's 3-element interferometer and the Berkeley Radio Astronomy Laboratory's Hat Creek interferometer. The Barrett committee saw the opportunity, and pointed to the need, for a national synthesis array for millimeter-wavelength astronomy. The array, they noted, should have the following properties:

(1) 1 arcsecond resolution at $115 \mathrm{GHz}$;

(2) $1000-2000 \mathrm{~m}^{2}$ total collecting area;

(3) good imaging capability at $\lambda 1 \mathrm{~mm}$.

Design of an array intended to fulfill these needs was begun at the NRAO in 1984. The resultant conceptual design was presented to a scientific workshop of approximately fifty astronomers held in Green Bank in September 1985. The strong scientific endorsement given for the millimeter-wave array (MMA) at this workshop and at other MMA design meetings held subsequently, placed particular emphasis on the array's imaging capability. The MMA should be a true imaging array. It should combine the brightness sensitivity of a single dish with the angular resolution of an array telescope. Frequently the objects to be observed are many times larger than the telescope beamwidth; this means that many overlapping regions must be observed and the individual images merged into a single mosaic image. Such mosaics can be constructed only if the array is fast and has good "snapshot" imaging capability (i.e. good instantaneous uvcoverage). Many antennas are required to maximize this capability. Finally, the MMA study groups have noted that new science, not possible with any existing instrument, will follow if the MMA is provided with a sensitive high frequency capability (to as high as $350 \mathrm{GHz}$ ) and sub-arcsecond resolution. 
TABLE I The Millimeter Array

\begin{tabular}{cll}
\hline Array- & \\
& Number of Antennas: & 40 \\
Total collecting area: & $2010 \mathrm{~m}^{2}$ \\
Angular Resolution & $0 . \lambda^{\prime} \lambda_{m m}$ \\
Antennas- & $8.0 \mathrm{~m}$ \\
Diameter: & $\lambda / 40$ at $1 \mathrm{~mm}$ \\
Precision: & $1 / 20$ beamwidth \\
Pointing: & \\
Transportable & $70 \mathrm{~m}$ \\
Configurations- & $250 \mathrm{~m}, 900 \mathrm{~m}$ \\
Compact: & $3 \mathrm{~km}$ \\
Intermediate (2): & $195-366 \mathrm{GHz}$ \\
High Resolution: & $30-50 \mathrm{GHz}$ \\
Frequencies- & $68-115 \mathrm{GHz}$ \\
Emphasis on: & $130-170 \mathrm{GHz}$ \\
Broadband Coverage: & Simultaneous multi-band \\
&
\end{tabular}

High Altitude, suitable for precision imaging at $1 \mathrm{~mm}$

\section{MMA OVERVIEW}

Translation of the scientific requirements into the parameters of the array has been the goal of the MMA design work over the last six years. Considerations which led to their definition include the following. The total collecting area of the MMA should exceed that of any existing millimeter-wave single dish. The array should be reconfigurable, as is the VLA, so as to permit study of both extended and compact emission regions with proper uv- sampling. Four scaled configurations of dimension $70 \mathrm{~m}, 250 \mathrm{~m}, 900 \mathrm{~m}$, and $3000 \mathrm{~m}$ are planned. The maximum extent of the array, $3 \mathrm{~km}$, corresponds to $0 . " 1$ angular resolution at $230 \mathrm{GHz}$. Finally, all antennas will be equipped with total-power systems so that the full collecting area of the MMA can be used as a single dish for those observations requiring this capability. The result is a versatile imaging instrument which emphasizes the following capabilities:

- Sub-arcsecond imaging at $115 \mathrm{GHz}$ and higher frequencies;

- Wide-field imaging, mosaicing;

- Rapid imaging, "snapshots" of high fidelity;

- Sensitive imaging at high frequency (>350 GHz);

- Simultaneous broadband, multi-band operation;

- Comprehensize 'single dish' capabilities. 
Together these properties define a unique instrument. High sensitivity implies that the total collecting area of all the individual elements in the array should be made as large as possible, while fast imaging is achieved by distributing the collecting area over many elements. Sub-arcsecond imaging places a constraint on the array dimension. Sensitive imaging at high frequency demands that the MMA be located on a high-altitude site with excellent atmospheric transparency.

\section{IMAGING AT MILLIMETER WAVELENGTHS}

The Millimeter Array combines the sensitivity provided by the collecting area of a telescope fifty meters in diameter with angular resolution, $<0 . " 1$, equaling that of the Hubble Space Telescope and operates at frequencies at which thermal emission processes illuminate the sky. This unprecedented combination of sensitivity and angular resolution at short wavelengths, together with the ability to image the spatial complexity of the sky at millimeter wavelengths, will make available for astronomical investigation a wealth of unique opportunities and new science. Scientists using the MMA will:

- Image the redshifted dust continuum emission from evolving galaxies at epochs of formation as early as $\mathrm{z}=10$;

- Reveal the kinematics of optically obscured galactic nuclei and QSOs on spatial scales smaller than $100 \mathrm{pc}$;

- Assess the influence that chemical and isotopic gradients in galactic disks have on star formation and spiral structure;

- Image heavily obscured regions containing protostars, and protostellar and pre-planetary disks in nearby molecular clouds, with a spatial resolution of 10 $\mathrm{AU}$ and kinematic resolution $<1 \mathrm{~km} / \mathrm{s}$.

- Detect the photospheric emission from hundreds of nearby stars in every part of the H-R diagram;

- Reveal the crucial isotopic and chemical gradients within circumstellar shells that reflect the chronology of stellar nuclear processing and envelope convection;

- Resolve the dust-formation region and probe the structure of the magnetic field in stellar winds;

- Obtain unobscured sub-arcsecond images of cometary nuclei, hundreds of asteroids, planetary atmospheres and surfaces, and solar regions of active particle acceleration.

\section{THE MILLIMETER ARRAY PROJECT}

Design work on the MMA has progressed for the last six years in the areas summarized below:

MMA Site The scientific requirements for the MMA include provision for imaging at sub-arcsecond angular resolution at the principal frequency bands of the MMA. The MMA must be reconfigurable, the antennas transportable, and the site big enough, and flat enough, to permit such reconfiguration. The 
site must also be at high altitude to realize the sky trnasparency needed for precision imaging at $200-350 \mathrm{GHz}$.

Three continental sites at elevations greater than 9000 feet at latitudes south of 36 degrees with an extent as large as $3 \mathrm{~km}$ are attractive for the MMA: (1) a plateau near Springerville, AZ; (2) an open pasture near Alpine, AZ; and (3) a site on the grounds of the Langmuir Laboratory in the Magdalena Mountains, NM.

Direct in situ measurements of the atmospheric transparency and atmospheric stability of the sites are being made. We have developed a 225 $\mathrm{GHz}$ tipping radiometer which measures atmospheric transparency every 10 minutes; once every 10 hours it takes a 1-hour total power scan at the zenith to measure the fluctuation spectrum of the atmospheric emission. The radiometer runs continuously, unattended. From these data, and ancillary considerations, a site selection will be made.

MMA Antenna and Telescope Optics Since the $350 \mathrm{GHz}$ atmospheric window is one of the two frequency bands given particular emphasis by the MMA (the other being the 1-mm band, 200-300 GHz) the antennas must support precision operation at this frequency. Specification of a 25-micron rss surface accuracy implies a gain reduction at $345 \mathrm{GHz}$ relative to the efficiency at lower frequencies of a little over $10 \%$, which gives the array high performance in this atmospheric window.

The working antenna design is a combination steel/CFRP antenna. It is a conventional Cassegrain except that the secondary focus is brought right down into the plinth (Coude focus). The receivers are located in fixed positions in the base of the antenna, a design feature which offers the following advantages: (1) the receivers are easily accessible; (2) the telescope balance is not affected by removing or installing receivers; and (3) there is no effect on the pointing from the drag of cables or compressor lines.

MMA Electronics Substantial progress has been made in the design of receivers for the MMA. At the lowest frequencies, $30-50 \mathrm{GHz}$, wideband HEMT amplifiers have been developed at the NRAO Central Development Laboratory with nearly $10 \mathrm{GHz}$ of bandwidth and a noise temperature less than $30 \mathrm{~K}$. At higher frequencies, the MMA will use low-noise SIS receivers. Current SIS receivers in use at the NRAO and elsewhere are narrow-band devices which, if used on the MMA would impose an unfortunate restriction on the desired MMA frequency agility. To circumvent this difficulty, we have developed an experimental fully integrated $3 \mathrm{~mm}$ SIS mixer with an instantaneous $50 \%$ bandwidth: it covers $70-115 \mathrm{GHz}$ with no tuning adjustments. The MMA will need such a broadband SIS mixer not only for $70-115 \mathrm{GHz}$, but also $130-170$ $\mathrm{GHz}, 200-300 \mathrm{GHz}$, and $310-350 \mathrm{GHz}$. Development work in these areas will be active in the next 5 years.

The MMA LO and IF signals will be transmitted by fiber-optics, the technology for which is in place at the Australia Telescope and will be implemented at the NRAO on the Green Bank Telescope. The local oscillators for the MMA will likely be varactor-diode frequency multipliers driven by phased-locked Gunn oscillators. Commercial development of InP Gunn diodes is continuing, but development of very widely tunable (25-40\% for the MMA) oscillators using these diodes will be undertaken as part of the MMA project. 
The design of the MMA correlator leans heavily on the design of the BIMA correlator for its flexible analog electronics and on the VLBA for the organization ("FX") of its digital correlation electronics. The correlator will process $2 \mathrm{GHz}$ bandwidth from each of 40 antennas. It computes the complex cross-power spectrum on each of 780 simultaneous interferometer baselines.

The $2 \mathrm{GHz}$ bandwidth processed by the correlator may be organized as desired by the astronomer.

\section{CONSTRUCTION COSTS AND SCHEDULE}

The proposal for construction of the MMA submitted by Associated Universities, Inc., to the NSF in July 1990 estimates the construction cost as $\$ 120 \mathrm{M}$ (1990 dollars) including a $15 \%$ contingency. The proposal requests significant design funding in 1992 and 1993 followed by major construction beginning in 1994. The array should be complete before the end of the decade. 